\title{
Serologic status and vaccine response against hepatitis $B$ virus after allogeneic hematopoietic cell transplantation in pediatric patients
}

\author{
Eui Soo Lee, ${ }^{1}$ Seong koo Kim, ${ }^{1,2}$ Seung Beom Han, ${ }^{1,3}$ Jae Wook Lee, ${ }^{1,2}$ Nack-Gyun Chung, ${ }^{1,2}$ \\ Bin Cho, ${ }^{1,2}$ Dae Chul Jeong, ${ }^{1,3}$ Jin Han Kangl,3
}

\begin{abstract}
Background: Although vaccination against hepatitis B virus (HBV) is recommended for hematopoietic cell transplantation (HCT) recipients, previous studies evaluating serologic status and immunologic response to $\mathrm{HBV}$ vaccination in pediatric allogeneic HCT recipients are not enough.
\end{abstract}

Objective: This study aimed to evaluate serologic status against HBV and immunologic responses to HBV vaccination in children and adolescents receiving allogeneic HCTs.

Methods: Medical records of the enrolled 61 pediatric patients $<19$ years of age who received their first allogeneic HCTs were retrospectively reviewed.

Results: Twenty-two (36.1\%) of the enrolled patients were positive for hepatitis B surface antibody (HBsAb) after HCT. Chronic graft-versus-host disease was significantly associated with negative HBsAb status after HCT $(p=0.01)$. With one dose of HBV vaccination after HCT, $40.5 \%$ of the vaccinated patients became positive for HBsAb. No clinical factor was associated with the positive conversion of $\mathrm{HBsAb}$ after vaccination.

Conclusions: Considering the unsatisfactory seropositive rate and vaccine response against HBV and the lack of significant clinical and laboratory factors predicting serostatus in HCT recipients, universal three doses of HBV vaccination should be necessary after allogeneic HCT.

Key words: hepatitis B virus, stem cell transplantation, leukemia, vaccination, child

\section{Citation:}

Lee, E. S., Kim, S. K., Han, S. B., Lee, J. W., Chung, N. G., Cho, B., Jeong, D. C., Kang, J. H. (2023) Serologic status and vaccine response against hepatitis B virus after allogeneic hematopoietic cell transplantation in pediatric patients. Asian Pac J Allergy Immunol, 41(1), 80-88. https://doi.org/10.12932/ap-221219-0724

\section{Introduction}

The advancement of hematopoietic cell transplantation (HCT) techniques and conservative care has improved the survival of HCT recipients. ${ }^{1}$ Especially in children expected to live for a longer time after HCT than adults, long-term care for improving the quality of life after HCT should be emphasized. Therefore, clinicians should make an effort to prevent community-acquired infection after HCT, and vaccination is one of the most effective strategies for preventing infection. Hepatitis $B$ virus (HBV) infection is one of major vaccine-preventable diseases, and its worldwide prevalence has been decreasing with the introduction of infantile $\mathrm{HBV}$ vaccination. ${ }^{2}$ Korea has also become a low intermediate $\mathrm{HBV}$ endemic area from being a previously high HBV endemic area after the inclusion of HBV vaccination in the National Immunization Program (NIP). ${ }^{3}$

Corresponding author:

Seung Beom Han

Department of Pediatrics, Daejeon St. Mary's Hospital,

College of Medicine, The Catholic University of Korea,

64, Daeheung-ro, Jung-gu, Daejeon 34943, Korea

E-mail: beomsid@catholic.ac.kr 
However, long-term survivors after HCT can travel to high $\mathrm{HBV}$ endemic areas and may participate in $\mathrm{HBV}$ infection-prone activities. Therefore, HBV vaccination should not be ignored in HCT recipients even though they live in low HBV endemic areas.

The 2013 Clinical Practice Guideline for Vaccination of the Immunocompromised Host of the Infectious Diseases Society of America (IDSA) states that HCT recipients should be considered "never vaccinated" like neonates, and therefore, should receive most of the vaccines administered to infants and young children after HCT regardless of their ages. ${ }^{4}$ Accordingly, three doses of hepatitis B virus (HBV) vaccines are recommended for HCT recipients. ${ }^{4}$ However, a recent guideline of the 2017 European Conference on Infections in Leukaemia (ECIL7) for vaccination of HCT recipients recommends three doses of $\mathrm{HBV}$ vaccination after HCT based on the result of post-HCT serologic tests against $\mathrm{HBV}^{5}{ }^{5}$ which is different from the IDSA recommendation. Furthermore, previous studies evaluating serologic status and immunologic and clinical response to $\mathrm{HBV}$ vaccination in $\mathrm{HCT}$ recipients that can support the necessity of $\mathrm{HBV}$ vaccination after HCT are not enough. ${ }^{6-8}$

In our hospital, HBV vaccination after HCT has been decided based on the results of hepatitis B surface antibody (HBsAb) testing performed after HCT. In Korea, HBV vaccination was introduced in 1983, and was included in the NIP as mandatory vaccination in $1995 .{ }^{9}$ After then, the primary HBV vaccination (at birth, 1 month and 6 months of age) rate has maintained over 93\%. ${ }^{10}$ Therefore, almost all of the pediatric HCT recipients were assumed to acquire a protective immunity against $\mathrm{HBV}$ infection from primary vaccination series during their infancy. Accordingly, the HBV re-vaccination strategy for healthy hosts, who were negative for $\mathrm{HBs} \mathrm{Bb}$ despite previous reception of primary HBV vaccination series, has been applied for pediatric HCT recipients in our hospital, without a consideration of immunosuppression effects of HCT. As a result, HBV vaccination was completed with a single dose administration in HCT recipients who exhibited positive seroconversion with one dose of $\mathrm{HBV}$ vaccination after $\mathrm{HCT}$, and three doses of $\mathrm{HBV}$ vaccination was administered to HCT recipients who were still negative for $\mathrm{HBsAb}$ after one dose of vaccination after HCT. This study was performed to evaluate the appropriateness of our vaccination strategy and to establish a proper vaccination strategy for pediatric HCT recipients. Serologic status against $\mathrm{HBV}$ was evaluated in children and adolescents receiving allogeneic HCTs, and immunologic responses to HBV vaccination after HCT were determined.

\section{Materials and methods \\ Patients}

Among children and adolescents aged less than 19 years old who received allogeneic HCTs in Seoul St. Mary's Hospital, Seoul, Korea, 105 patients were requested to infectious disease physicians for vaccination after HCT between January 2015 and June 2018. Of the 105 patients, 69 patients, whose HBsAb titers were measured using a quantitative electrochemiluminescence immunoassay (ECLIA) test, were included in this study. Those whose $\mathrm{HBsAb}$ tests were performed using radioimmunoassay were excluded. Excluding eight patients who received more than one HCT, the remaining 61 patients receiving their first HCTs were finally enrolled in this study (Figure 1). In our hospital, HCT recipients are requested for vaccination when one year or more has passed since their allogeneic HCTs and immune suppression therapy has finished with controlled graft-versus-host diseases (GvHDs). For GvHD prophylaxis after allogeneic HCT, cyclosporine was administered from day -1 , and four doses of intravenous methotrexate $\left(5 \mathrm{mg} / \mathrm{m}^{2}\right)$ were administered on days $1,3,6$, and 11. Intravenous immunoglobulin (IVIG, $400-500 \mathrm{mg} / \mathrm{kg}$ ) was administered six times every 2 weeks from 1 week after HCT, and then six times monthly. IVIG replacement was discontinued when renal dysfunction occurred. This study was approved by the Institutional Review Board of Seoul St. Mary's Hospital with waiver for acquiring informed consent (Approval number: KC18RESI0503).

\section{Data collection and analysis}

Medical records of the enrolled patients were retrospectively reviewed. Demographic data, including sex and ages when underlying hematologic/oncologic diseases were diagnosed and when the first HBsAb test after HCT was performed, were investigated. Clinical data, such as underlying hematologic/oncologic disease, donor type, hematopoietic cell source, pre-HCT conditioning regimen, and presence of acute and chronic GvHDs, were gathered. In addition, time intervals from HCT, from the completion of immune suppression, and from the last dose of IVIG supplement, to the first HBsAb test after HCT were determined. HBsAb status of the HCT donor, pre- and post-HCT HBsAb titers of the HCT recipient, white blood cell (WBC), neutrophil, lymphocyte, and monocyte counts on the first HBsAb test after HCT, and lymphocyte subset count and immunoglobulin levels at one year after HCT were investigated as laboratory data. HBsAb titers were measured using a commercial ECLIA kit (Elecsys Anti-HBs, Roche Diagnostics GmBH, Mannheim, Germany). The measuring range of this ECLIA kit was from $2.00 \mathrm{IU} / \mathrm{L}$ to $1,000.00$ $\mathrm{IU} / \mathrm{L}$, and therefore, the measured HBsAb titer $<2.00 \mathrm{IU} / \mathrm{L}$ and $>1,000.00 \mathrm{IU} / \mathrm{L}$ were regarded as $1.00 \mathrm{IU} / \mathrm{L}$ and $1,000.00 \mathrm{IU} / \mathrm{L}$, respectively. HBsAb titers $\geq 10.00 \mathrm{IU} / \mathrm{L}$ and $<10.00 \mathrm{IU} / \mathrm{L}$ were defined as positive and negative results, respectively. 


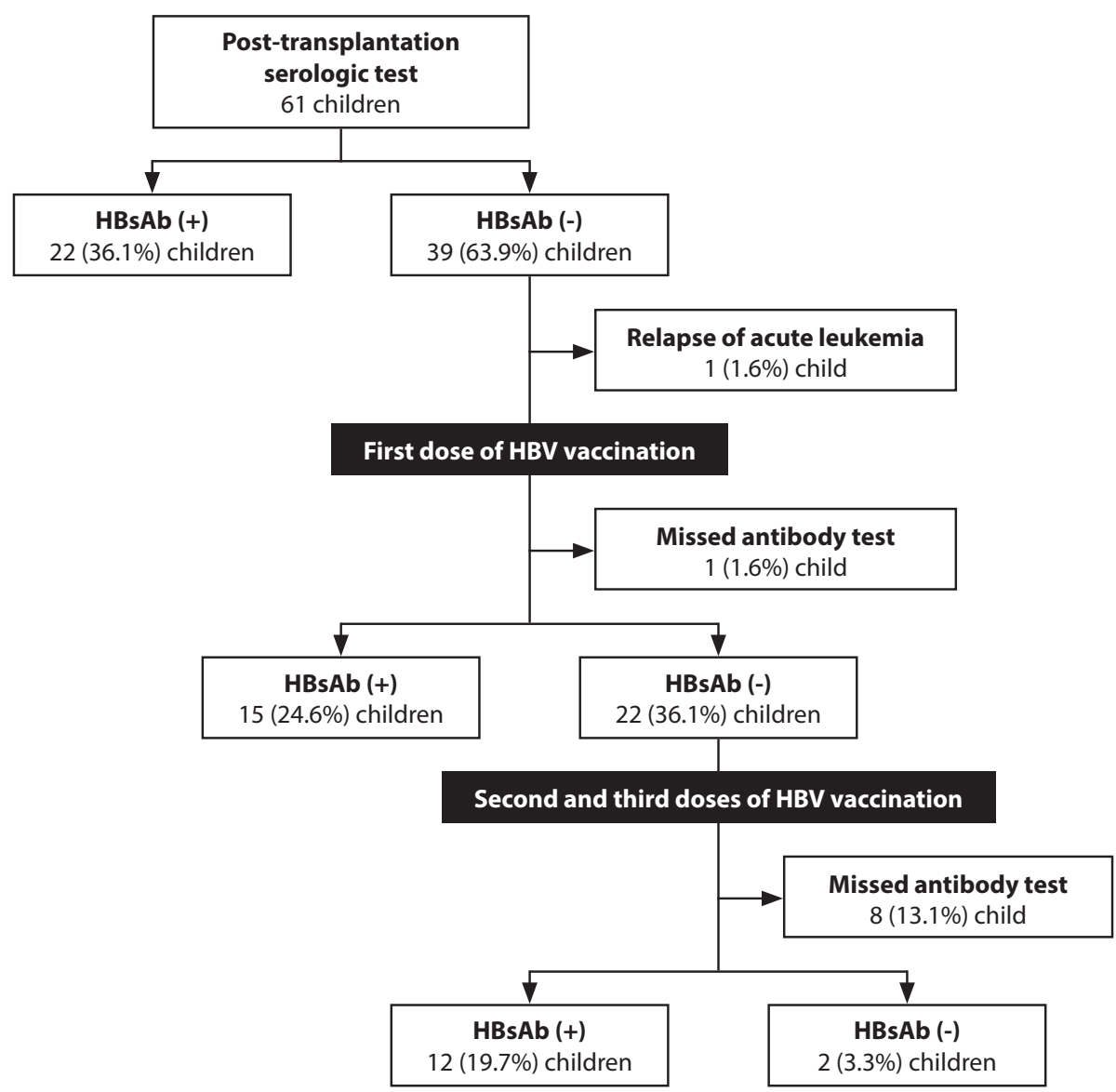

Figure 1. Summary of the study results.

The enrolled patients were divided into $\mathrm{HBs} A \mathrm{~b}$ positive and negative groups based on the first $\mathrm{HBsAb}$ results after HCT. Investigated data were compared between the two patient groups to find significant factors for $\mathrm{HBsAb}$ positivity after HCT. If HBsAb was negative after HCT, one dose of HBV vaccination was administered to the HCT recipient. $\mathrm{HBs} A \mathrm{~b}$ tests were repeated at least 4 weeks after vaccination. Patients were divided into $\mathrm{HBs} A b$ positive and negative groups again based on $\mathrm{HBs} A b$ results after $\mathrm{HBV}$ vaccination, and investigated data were compared between the two patient groups to determine significant factors for immunologic response to HBV vaccination after HCT.

\section{Statistical analysis}

For comparisons between patient groups, continuous and categorical data were compared using Mann-Whitney and Fisher's exact tests, respectively. The SPSS 21 program (IBM Corporation, Armonk, NY, USA) was used for statistical analyses, and statistical significance was defined as a $p$-value $<0.05$.

\section{Results \\ HBs Ab status after HCT}

Thirty-one $(50.8 \%)$ of the enrolled 61 patients were males. The median age on the diagnosis of underlying hematologic/oncologic disease was 5 years (range, 0-17), and that on the HBsAb test after HCT was 8 years (range, 2-19). None of the enrolled patients were positive for hepatitis B surface antigen before and after HCT. HBsAb testing was performed a median of 14 months (range, 12-31) after HCT, a median of 5 months (range, 0-14) after completing immune suppression therapy, and a median of 6 months (range, 3-29) after the last administration of IVIG. HBsAb after HCT was positive in $22(36.1 \%)$ patients (Table 1, Figure 1). Underlying disease, donor type, hematopoietic cell source, pre-HCT conditioning regimen, and positive donor $\mathrm{HBsAb}$ were not significantly associated with positive HBsAb after HCT (Table 1). Chemotherapy for underlying disease preceding HCT might influence on the loss of HBsAb before HCT; however, positive HBsAb rates after HCT were not significantly different between patients with underlying diseases requiring chemotherapy (leukemia, lymphoma, myelodysplastic syndrome, and hemophagocytic lymphohistiocytosis) and those with underlying diseases not requiring chemotherapy (severe aplastic anemia and Wiskott-Aldrich syndrome) $(34.8 \%$ vs $40.0 \%, p=0.76)$. Only chronic GvHD occurred more frequently in the HBsAb negative group than in the HBsAb positive group $(p=0.01$, Table 1). 
Table 1. Comparison between patients with negative and positive results for HBsAb after allogeneic HCT.

\begin{tabular}{|c|c|c|c|}
\hline Factor & $\begin{array}{l}\text { HBsAb negative } \\
\qquad(\mathbf{N}=39)\end{array}$ & $\begin{array}{l}\text { HBsAb positive } \\
\qquad(\mathrm{N}=22)\end{array}$ & $\boldsymbol{P}$ \\
\hline Male sex & $22(56.4)$ & $9(40.9)$ & 0.29 \\
\hline Age on the diagnosis of underlying disease (year) & $5(0-17)$ & $6(0-16)$ & 0.72 \\
\hline Age on the first HBsAb test after HCT (year) & $8(2-19)$ & $8(2-18)$ & 0.46 \\
\hline Underlying hematologic/oncologic diseases & & & 0.76 \\
\hline Acute lymphoblastic leukemia & $13(33.3)$ & $5(22.7)$ & \\
\hline Acute myeloid leukemia & $9(23.1)$ & $7(31.8)$ & \\
\hline Other leukemias & $2(5.1)$ & $2(9.1)$ & \\
\hline Lymphoma & $1(2.6)$ & $0(0.0)$ & \\
\hline Myelodysplastic syndrome & $4(10.3)$ & $2(9.1)$ & \\
\hline Severe aplastic anemia & $9(23.1)$ & $5(22.7)$ & \\
\hline Hemophagocytic lymphohistiocytosis & $1(2.6)$ & $0(0.0)$ & \\
\hline Wiskott-Aldrich syndrome & $0(0.0)$ & $1(4.5)$ & \\
\hline Positive donor $\mathrm{HBsAb}^{*}$ & $33(91.7)$ & $21(95.5)$ & 1.00 \\
\hline HCT donor type ${ }^{a}$ & & & 0.30 \\
\hline Matched familial donor & $8(22.2)$ & $2(9.1)$ & \\
\hline Matched unrelated donor & $20(55.6)$ & $13(59.1)$ & \\
\hline Mismatched familial donor & $5(13.9)$ & $2(9.1)$ & \\
\hline Mismatched unrelated donor & $3(8.3)$ & $5(22.7)$ & \\
\hline Hematopoietic cell source & & & 0.23 \\
\hline Bone marrow & $7(17.9)$ & $2(9.1)$ & \\
\hline Peripheral blood & $29(74.4)$ & $20(90.9)$ & \\
\hline Umbilical cord blood & $3(7.7)$ & $0(0.0)$ & \\
\hline Pre-HCT conditioning therapy & & & 0.79 \\
\hline Myeloablative conditioning & $25(64.1)$ & $15(68.2)$ & \\
\hline Non-myeloablative conditioning & $14(35.9)$ & $7(31.8)$ & \\
\hline Anti-thymocyte globulin use & $31(79.5)$ & $20(90.9)$ & 0.31 \\
\hline Acute graft-versus-host disease & $25(64.1)$ & $11(50.0)$ & 0.42 \\
\hline Chronic graft-versus-host disease $^{\dagger}$ & $13(34.2)$ & $1(4.5)$ & 0.01 \\
\hline Months from HCT to HBsAb test & $14(12-31)$ & $14(12-20)$ & 0.97 \\
\hline Months from last dose of IVIG to HBsAb test & $6(3-29)$ & $6(4-11)$ & 0.83 \\
\hline $\begin{array}{l}\text { Months from the completion of immune suppression } \\
\text { therapy to HBsAb test }\end{array}$ & $5(0-14)$ & $5(0-9)$ & 0.73 \\
\hline Pre-HCT positive $\mathrm{HBsAb} \ddagger$ & $17(63.0)$ & $9(90.0)$ & 0.22 \\
\hline \multicolumn{4}{|l|}{ Blood cell count on the first HBsAb test $\left(\right.$ cells $\left./ \mathrm{mm}^{3}\right)$} \\
\hline White blood cell count & $6,280(1,280-11,600)$ & $7,100(4,240-13,920)$ & 0.39 \\
\hline Absolute neutrophil count & $2,638(307-5,554)$ & $3,234(1,594-7,795)$ & 0.30 \\
\hline Absolute lymphocyte count & $2,749(589-7,076)$ & $2,819(1,551-5,707)$ & 0.44 \\
\hline
\end{tabular}


Table 1. (Continued)

\begin{tabular}{|c|c|c|c|}
\hline Factor & $\begin{array}{l}\text { HBsAb negative } \\
\qquad(\mathrm{N}=39)\end{array}$ & $\begin{array}{l}\text { HBsAb positive } \\
\qquad(\mathrm{N}=\mathbf{2 2})\end{array}$ & $\boldsymbol{P}$ \\
\hline \multicolumn{4}{|c|}{ Lymphocyte subset count at 1 year after HCT $\left(\text { cells } / \mathrm{mm}^{3}\right)^{\varsigma}$} \\
\hline Absolute lymphocyte count & $2,352(284-8,935)$ & $2,699(1,032-6,938)$ & 0.23 \\
\hline $\mathrm{CD} 3+$ cell count & $1,574(220-5,450)$ & $1,666(636-4,468)$ & 0.41 \\
\hline CD4+ cell count & $575(82-2,469)$ & $629(176-1,238)$ & 0.81 \\
\hline CD8+ cell count & $709(98-3,558)$ & $907(281-3,254)$ & 0.36 \\
\hline CD19+ cell count & $391(1-1,835)$ & $471(59-1,603)$ & 0.88 \\
\hline CD56+ cell count & $155(27-1,608)$ & $330(45-788)$ & $<0.01$ \\
\hline \multicolumn{4}{|c|}{ Immunoglobulin levels at 1 year after $\mathrm{HCT}(\mathrm{mg} / \mathrm{dL})^{\varsigma}$} \\
\hline IgG & $948(385-1,669)$ & $989(497-1,268)$ & 0.78 \\
\hline IgM & $80(2-460)$ & $94(22-184)$ & 0.32 \\
\hline $\operatorname{IgA}$ & $64(12-218)$ & $79(26-272)$ & 0.46 \\
\hline $\operatorname{IgE}$ & $53(1-3,572)$ & $195(1-2,370)$ & 0.11 \\
\hline
\end{tabular}

Values are presented as number (\%) or median (range).

${ }^{*}$ Donor HBsAb status and donor type could not be specified in three patients receiving cord blood transplantation in the HBsAb negative group.

${ }^{\dagger}$ The development of chronic graft-versus-host diseases could not be determined in one patient in the HBsAb negative group.

${ }^{*}$ Pre-HCT HBsAb status was identified in 27 patients in the HBsAb negative group and 10 patients in the HBsAb positive group.

${ }^{9}$ Lymphocyte subset counts and immunoglobulin levels at 1 year after HCT were not tested in one patient in the HBsAb negative group.

HBsAb, hepatitis B surface antibody; HCT, hematopoietic cell transplantation; IVIG, intravenous immunoglobulin

Pre-HCT HBsAb status was identified in 37 (60.7\%) patients. All the HBsAb tests were performed on the diagnosis of underlying disease rather than immediately before HCT. Among them, $26(70.3 \%)$ patients were positive for HBsAb before HCT, and nine (34.6\%) of them remained positive for HBsAb after HCT. One (9.1\%) of 11 patients who were negative for $\mathrm{HBsAb}$ before $\mathrm{HCT}$ became positive for HBsAb after HCT. For this patient, donor HBsAb was positive. The presence of HBsAb before HCT was not significantly associated with positive HBsAb after HCT ( $p=0.22)$; however, the median HBsAb titer before HCT (361.50 IU/L; range, 4.13-1,000.00) of patients with positive HBsAb after HCT was significantly higher than that (25.48 IU/L; range, $1.00-549.80)$ of those with negative HBsAb after HCT $(p=0.01)$.

\section{Immunologic response to $\mathrm{HBV}$ vaccination after $\mathrm{HCT}$}

Among the 39 patients with negative HBsAb after HCT, 38 patients received one dose of HBV vaccination (Figure 1). The remaining one patient was diagnosed with relapse of underlying acute myeloid leukemia after the HBsAb test. $\mathrm{HBsAb}$ tests were repeated in 37 of the vaccinated 38 patients. Among the 37 patients, 15 (40.5\%) patients became positive for HBsAb (Figure 1). No clinical factors were significantly associated with a positive response to $\mathrm{HBV}$ vaccination after HCT (Table 2). The presence of HBsAb before HCT was not significantly associated with a positive conversion of $\mathrm{HBsAb}$ after $\mathrm{HBV}$ vaccination $(p=1.00)$, and the median $\mathrm{HBsAb}$ titer before HCT (13.83 IU/L; range, 1.00-205.30) of patients with positive $\mathrm{HBsAb}$ after $\mathrm{HBV}$ vaccination was not significantly different from that $(28.07 \mathrm{IU} / \mathrm{L} ;$ range, 1.00-549.80) of those with negative HBsAb after vaccination $(p=0.78)$. In addition, the median HBsAb titers measured between HCT and HBV vaccination were also not significantly different between patients with positive and negative $\mathrm{HBs} A b s$ after $\mathrm{HBV}$ vaccination (1.00 IU/L [range, $1.00-8.50$ ] vs $1.00 \mathrm{IU} / \mathrm{L}$ [range, 1.00-9.31]).

The WBC $(p=0.02)$ and absolute neutrophil $(p=0.04)$ counts determined on the day of first HBsAb test after HCT were significantly higher in patients who did not respond to HBV vaccination after HCT than in those who responded to HBV vaccination after HCT (Table 2). The WBC and lymphocyte subset counts determined at 1 year after HCT were also higher in patients who did not respond to HBV vaccination after HCT than in those who responded to HBV vaccination, although it was not statistically significant (Table 2).

All the 22 patients with negative HBsAb after one dose of $\mathrm{HBV}$ vaccination completed three doses of $\mathrm{HBV}$ vaccination schedule (Figure 1). In eight patients of them, HBsAb status could not be determined after three doses of vaccination because HBsAb testing was not ordered. The remaining 14 patients repeated $\mathrm{HBs} A b$ tests after the third dose of $\mathrm{HBV}$ vaccination, and $12(85.7 \%)$ of them became positive for HBsAb (Figure 1). The two seronegative patients received an additional one dose of HBV vaccination 6 and 13 months after their third vaccination, respectively, and became positive for HBsAb. 
Table 2. Comparison between patients with negative and positive results for $\mathrm{HBs} \mathrm{Ab}$ after one dose of $\mathrm{HBV}$ vaccination.

\begin{tabular}{|c|c|c|c|}
\hline Factor & $\begin{array}{l}\text { HBsAb negative } \\
\qquad(\mathbf{N}=22)\end{array}$ & $\begin{array}{l}\text { HBsAb positive } \\
\qquad(\mathbf{N}=15)\end{array}$ & $\boldsymbol{P}$ \\
\hline Male sex & $13(59.1)$ & $8(53.3)$ & 0.75 \\
\hline Age on the diagnosis of underlying disease (year) & $6(0-17)$ & $5(2-15)$ & 0.70 \\
\hline Age on the first HBsAb test after HCT (year) & $10(2-19)$ & $7(3-18)$ & 0.64 \\
\hline Underlying hematologic/oncologic disease & & & 0.39 \\
\hline Acute lymphoblastic leukemia & $9(40.9)$ & $4(26.7)$ & \\
\hline Acute myeloid leukemia & $4(18.2)$ & $4(26.7)$ & \\
\hline Other leukemias & $2(9.1)$ & $0(0.0)$ & \\
\hline Lymphoma & $1(4.5)$ & $0(0.0)$ & \\
\hline Myelodysplastic syndrome & $1(4.5)$ & $3(20.0)$ & \\
\hline Severe aplastic anemia & $5(22.7)$ & $3(20.0)$ & \\
\hline Hemophagocytic lymphohistiocytosis & $0(0.0)$ & $1(6.7)$ & \\
\hline Positive donor $\mathrm{HBsAb}^{*}$ & $16(84.2)$ & $15(100.0)$ & 0.24 \\
\hline HCT donor type ${ }^{*}$ & & & 0.70 \\
\hline Matched familial donor & $5(26.3)$ & $3(20.0)$ & \\
\hline Matched unrelated donor & $9(47.4)$ & $10(66.7)$ & \\
\hline Mismatched familial donor & $3(15.8)$ & $1(6.7)$ & \\
\hline Mismatched unrelated donor & $2(10.5)$ & $1(6.7)$ & \\
\hline Hematopoietic cell source & & & 0.21 \\
\hline Bone marrow & $5(22.7)$ & $2(13.3)$ & \\
\hline Peripheral blood & $14(64.6)$ & $13(86.7)$ & \\
\hline Umbilical cord blood & $3(13.6)$ & $0(0.0)$ & \\
\hline Pre-HCT conditioning therapy & & & 0.49 \\
\hline Myeloablative conditioning & $16(72.7)$ & $9(60.0)$ & \\
\hline Non-myeloablative conditioning & $6(27.3)$ & $6(40.0)$ & \\
\hline Anti-thymocyte globulin use & $16(72.7)$ & $13(86.7)$ & 0.43 \\
\hline Acute graft-versus-host disease & $16(72.7)$ & $7(46.7)$ & 0.17 \\
\hline Chronic graft-versus-host disease & $7(31.8)$ & $5(35.7)$ & 1.00 \\
\hline Months from HCT to HBsAb test & $14(13-21)$ & $13(12-30)$ & 0.14 \\
\hline Months from HCT to HBV vaccination & $15(13-22)$ & $14(13-42)$ & 0.35 \\
\hline Months from last dose of IVIG to HBsAb test & $6(4-14)$ & $5(3-29)$ & 0.13 \\
\hline $\begin{array}{l}\text { Months from the completion of immune suppression } \\
\text { therapy to HBsAb test }\end{array}$ & $6(0-13)$ & $4(0-8)$ & 0.55 \\
\hline Pre-HCT positive $\mathrm{HBsAb}^{\dagger}$ & $12(63.2)$ & $5(71.4)$ & 1.00 \\
\hline \multicolumn{4}{|l|}{ Blood cell count on the first HBsAb test $\left(\right.$ cells $/ \mathrm{mm}^{3}$ ) } \\
\hline White blood cell count & $7,205(2,590-11,600)$ & $5,560(1,280-9,000)$ & 0.02 \\
\hline Absolute neutrophil count & $3,482(932-5,554)$ & $1,953(307-4,596)$ & 0.04 \\
\hline Absolute lymphocyte count & $3,046(1,114-7,076)$ & $2,164(589-4,950)$ & 0.07 \\
\hline
\end{tabular}


Table 2. (Continued)

\begin{tabular}{|l|c|c|c|}
\multicolumn{1}{|c|}{ Factor } & $\begin{array}{c}\text { HBsAb negative } \\
(\mathbf{N}=\mathbf{2 2})\end{array}$ & $\begin{array}{c}\text { HBsAb positive } \\
(\mathbf{N}=\mathbf{1 5})\end{array}$ & $P$ \\
\hline Lymphocyte subset count at 1 year after HCT (cells/mm3) & & & \\
Absolute lymphocyte count & $2,472(977-5,972)$ & $2,022(284-8,935)$ & 0.31 \\
CD3+ cell (\%) & $1,571(515-4,819)$ & $1,413(220-5,450)$ & 0.60 \\
CD4+ cell (\%) & $595(186-1,466)$ & $552(82-1,394)$ & 0.74 \\
CD8+ cell (\%) & $795(210-3,558)$ & $627(98-3,315)$ & 0.55 \\
CD19+ cell (\%) & $444(1-1,835)$ & $351(21-1,226)$ & 0.45 \\
CD56+ cell (\%) & $181(44-613)$ & $134(27-1,608)$ & 0.07 \\
Immunoglobulin levels 1 year after HCT (mg/dL) & & & 0.51 \\
IgG & $996(385-1,669)$ & $936(602-1,409)$ & 0.62 \\
IgM & $78(2-460)$ & $83(42-251)$ & 0.49 \\
IgA & $61(12-218)$ & $66(38-143)$ & 0.49 \\
\hline IgE & $56(1-3,056)$ & $56(8-3,572)$ & \\
\hline
\end{tabular}

Values are presented as number (\%) or median (range).

${ }^{\star}$ Donor HBsAb status and donor type could not be specified in three patients receiving cord blood transplantation in the HBsAb negative group. ${ }^{\dagger}$ Pre-HCT HBsAb status was identified in 19 patients in the HBsAb negative group and seven patients in the HBsAb positive group.

${ }^{*}$ Lymphocyte subset counts and immunoglobulin levels at 1 year after HCT were not tested in one patient in the HBsAb positive group.

HBsAb, hepatitis B surface antibody; HBV, hepatitis B virus; HCT, hematopoietic cell transplantation; IVIG, intravenous immunoglobulin.

\section{Discussion}

This study investigated serologic status against $\mathrm{HBV}$ in children and adolescents receiving allogeneic HCT. In addition, the immunologic response to $\mathrm{HBV}$ vaccination after HCT was also determined. About two-thirds of allogeneic HCT recipients were negative for $\mathrm{HBsAb}$ after HCT, and less than half of the patients responded to one dose of HBV vaccination after HCT.

Pre-HCT positive HBsAb rate in this study was $70.3 \%$, which is similar to the positive rate of $68.5 \%$ in healthy Korean children younger than 10 years of age. ${ }^{9}$ Park et al. reported that $69.1 \%$ of Korean adults who were HBsAb positive before allogeneic HCT remained positive for $\mathrm{HBsAb}$ after $\mathrm{HCT}^{11}$ whereas only $34.6 \%$ of pediatric patients who were $\mathrm{HBsAb}$ positive before allogeneic $\mathrm{HCT}$ remained $\mathrm{HBsAb}$ positive after HCT in this study. In Korea, HBV vaccination became mandatory in 1995, and a high vaccination rate has been maintained. ${ }^{10}$ Therefore, almost all patients enrolled in this study should acquire HBsAb through their primary HBV vaccination rather than natural HBV infection before HCT. However, $64.2 \%$ of the adult patients, with a median age of 35 years (range, 15-64), enrolled in the study of Park et al. were positive for hepatitis $\mathrm{B}$ core antibody, ${ }^{11}$ and therefore, most of them should acquire $\mathrm{HBs} A b$ through previous $\mathrm{HBV}$ infection. Previous reports showed higher seropositive rate and higher $\mathrm{HBs} A b$ titers after $\mathrm{HCT}$ in $\mathrm{HCT}$ recipients experiencing $\mathrm{HBV}$ infection than in those receiving $\mathrm{HBV}$ vaccination before HCT. ${ }^{6,12}$ Even in low $\mathrm{HBV}$ endemic areas, HBV vaccination after HCT should be encouraged because almost all HCT recipients acquire immunity against $\mathrm{HBV}$ through vaccination and they are more likely to lose the immunity than those experiencing natural HBV infection before HCT.

In our hospital, the positive $\mathrm{HBsAb}$ rate of pediatric patients underwent chemotherapy for leukemia without HCT was $14.6 \%{ }^{13}$ Although allogeneic HCT is considered to cause more potent immune suppression compared to chemotherapy, the positive $\mathrm{HBsAb}$ rate was higher in patients receiving allogeneic HCT in this study than in those receiving chemotherapy only $(p=0.17)$. This difference might be caused by positive donor HBsAb and IVIG administered after HCT, although the small number of enrolled patients in this study could not be ignored. Previous studies and this study showed no significant association between positive $\mathrm{HBsAb}$ status after HCT and positive donor $\mathrm{HBsAb},{ }^{6-8}$ and transferred donor $\mathrm{HBsAb}$ tended to decrease as time went on. ${ }^{14}$ Most of the patients enrolled in this study had received 12 times of IVIG replacement after HCT. Although the half-life of administered IVIG was reported as 21 days in HCT recipients, ${ }^{15}$ multiple IVIG administration could lead to accumulation of $\mathrm{HBsAb}$ and resultant increase of $\mathrm{HBsAb}$ titers in a portion of $\mathrm{HCT}$ recipients. Considering the insignificant difference of $\mathrm{HBs} \mathrm{Ab}$ positivity rates between patients receiving HCT and those underwent chemotherapy in our hospital, further studies including more patients not receiving routine IVIG replacement after HCT are necessary for determining the IVIG effect. 
Chronic GvHD was significantly associated with negative HBsAb status after HCT in this study, similar to previous studies. ${ }^{8,11}$ The CD56+ cell count was significantly higher in patients with positive HBsAb after HCT than in those with negative HBsAb after HCT in this study, while CD56+ cells do not participate in antibody production. ${ }^{16}$ Considering a significant association between a persistent low CD56+ cell count after HCT and development of chronic GvHD was previously reported, ${ }^{17,18}$ the lower CD56+ cell count might be associated with higher frequency of chronic GvHD in patients with negative HBsAb after HCT compared to those with positive HBsAb after HCT.

The positive conversion rate of $\mathrm{HBsAb}$ after boosting HBV vaccination in healthy hosts who previously received three doses of HBV vaccination was reported to be $88 \%{ }^{19}$ The positive conversion rate of $\mathrm{HBsAb}$ after one dose of HBV vaccination was $40.5 \%$ in this study; this was between the positive conversion rates in infants at $25 \%$ and in young adults at $30-55 \%$, who received the first dose of $\mathrm{HBV}$ vaccine in their life. ${ }^{19}$ Therefore, the positive conversion rate of $\mathrm{HBs} A \mathrm{~b}$ after one dose of HBV vaccination in this study seemed not to represent an anamnestic response to boosting vaccination, but to represent a response to the first dose among three doses of vaccination series in HCT recipients losing their protective immunity against HBV through HCT. If we consider that (1) a portion of positive $\mathrm{HBsAb}$ after HCT might be caused by repeated IVIG replacement therapy, (2) a low positive conversion rate of HBsAb after one dose of vaccination might represent the loss of protective immunity after HCT, and (3) the positive rate and titer of $\mathrm{HBsAb}$ had decreased over time since HCT in previous studies, ${ }^{8,11}$ a universal three doses of $\mathrm{HBV}$ vaccination seems to be adequate for HCT recipients. In addition, $14.3 \%$ of patients receiving three doses of $\mathrm{HBV}$ vaccination after $\mathrm{HCT}$ were still negative for $\mathrm{HBsAb}$, and therefore, $\mathrm{HBsAb}$ re-testing is necessary after completing three doses of vaccination in HCT recipients in consistent with the IDSA and ECIL7 guidelines. $^{4,5}$ In healthy hosts, the positive conversion of $\mathrm{HBs} \mathrm{Ab}$ after boosting $\mathrm{HBV}$ vaccination was significantly associated with the vaccinee's HBsAb titer before boosting vaccination. ${ }^{20}$ In this study, the median of $\mathrm{HBsAb}$ titers measured between HCT and HBV vaccination was not significantly different between patients with positive and negative $\mathrm{HBsAb}$ results after $\mathrm{HBV}$ vaccination. Further studies on the association between HBsAb titer after HCT and seroconversion rate after $\mathrm{HBV}$ vaccination may be helpful for establishing a strategy to decide the doses of $\mathrm{HBV}$ vaccination in $\mathrm{HCT}$ recipients based on their HBsAb titers.

The association between low CD4+ cell count and low response rate to $\mathrm{HBV}$ vaccination after $\mathrm{HCT}$ was previously reported. ${ }^{21}$ However, in this study, WBC and lymphocyte counts of patients who did not respond to HBV vaccination after HCT were higher than those of patients who responded to HBV vaccination, although it was not statistically significant. This discrepancy can represent the importance of the recovery of immune cell function and memory cell count rather than total immune cell count for an adequate immunologic response in HCT recipients. ${ }^{22-24}$
Therefore, appropriate strategies for post-HCT vaccination based on the recovery of naive and memory cell counts and the restoration of their function should be established in future studies.

This study had several limitations. The number of enrolled patients was small due to exclusion of patients in whom $\mathrm{HBsAb}$ testing was performed using radioimmunoassay. In addition, $39.3 \%$ of the enrolled patients had no data on pre-HCT HBsAb status. Even in those with pre-HCT $\mathrm{HBsAb}$ results, their $\mathrm{HBsAb}$ tests were performed on the diagnosis of underlying diseases rather than just before HCT. Therefore, the negative effect of chemotherapy and HCT on immunity against $\mathrm{HBV}$ could not be independently determined. In addition, the association between $\mathrm{HBsAb}$ titer measured just before HCT and immunologic response to HBV vaccination after HCT could not be determined. Pre-HCT HBV vaccination history was not identified in most of the enrolled patients and hepatitis B core antibodies were not tested. Therefore, the exact influence of pre-HCT HBV infection and vaccination on serologic status after HCT was not determined although few patients might be previously infected by $\mathrm{HBV}$ under a high $\mathrm{HBV}$ vaccination rate during infancy and low intermediate prevalence of $\mathrm{HBV}$ infection in Korea. To overcome these limitations, a well-designed prospective study establishing the type and time of serologic tests and identifying pre- and post-HCT vaccination history is necessary. Some of patients with positive $\mathrm{HBsAb}$ results after HCT or after one dose of HBV vaccination after HCT could have their own protective immunity, not derived from donor HBsAb or administered IVIG. These patients will exhibit an anamnestic response to boosting HBV vaccination several years after HCT. Therefore, future studies evaluating such anamnestic responses should be helpful for selecting vaccination candidates among HCT recipients.

In conclusion, this study confirmed the necessity of universal HBV vaccination after allogeneic HCT. Especially in countries where almost all children acquire HBV immunity through vaccination, post-HCT vaccination should be encouraged. Based on no existence of significant clinical and laboratory factors predicting seropositive status and vaccination response after HCT, uncertain origin of HBsAb after HCT or after one dose of HBV vaccination, and unsatisfactory positive conversion rate of $\mathrm{HBsAb}$ after one dose of $\mathrm{HBV}$ vaccination, three doses of $\mathrm{HBV}$ vaccination should be adequate for $\mathrm{HCT}$ recipients. In addition, $\mathrm{HBs} \mathrm{Ab}$ re-testing is necessary after three doses of vaccination.

\section{Conflict of interest}

There is no conflict of interest for all authors of this study.

\section{Author contributions}

- $\mathrm{SBH}, \mathrm{BC}$ and JHK conceptualized and designed this study.

- ESL, SKK and JWL collected the data.

- JWL, NGC and DCJ analyzed the data.

- ESL, SKK and SBH wrote the original manuscript.

- BC, NGC and JHK critically reviewed and revised the manuscript. 


\section{References}

1. Atilla E, Atilla PA, Toprak SK, Demirer T. A review of late complications of allogeneic hematopoietic stem cell transplantations. Clin Transplant. 2017;31:e13062.

2. World Health Organization. Hepatitis B vaccines. WHO position paper - July 2017. Wkly Epidemiol Rec. 2017;92:369-92.

3. Kim H, Shin AR, Chung HH, Kim MK, Lee JS, Shim JJ, et al. Recent trends in hepatitis B virus infection in the general Korean population. Korean J Intern Med. 2013;28:413-9.

4. Rubin LG, Levin MJ, Ljungman P, Davies EG, Avery R, Tomblyn M, et al. 2013 IDSA clinical practice guideline for vaccination of the immunocompromised host. Clin Infect Dis. 2014;58:e44-100.

5. Cordonnier C, Einarsdottir S, Cesaro S, Di Blasi R, Mikulska M, Rieger C, et al. Vaccination of haemopoietic stem cell transplant recipients: guidelines of the 2017 European Conference on Infections in Leukaemia (ECIL 7). Lancet Infect Dis. 2019;19:e200-12.

6. Idilman R, Ustun C, Karayalcin S, Aktemel A, Turkyilmaz AR, Ozcan M, et al. Hepatitis $B$ virus vaccination of recipients and donors of allogeneic peripheral blood stem cell transplantation. Clin Transplant. 2003;17: 438-43.

7. Jaffe D, Papadopoulos EB, Young JW, O'reilly RJ, Prockop S, Kernan NA, et al. Immunogenicity of recombinant hepatitis B vaccine (rHBV) in recipients of unrelated or related allogeneic hematopoietic cell (HC) transplants. Blood. 2006;108:2470-5.

8. Kaloyannidis P, Batsis I, Yannaki E, Adamidou D, Bartzoudis D, Papathanasiou $M$, et al. Allografted recipients immunized against hepatitis B virus are at high risk of gradual surface antibody (HbsAb) disappearance post transplant, regardless of adoptive immunity transfer. Biol Blood Marrow Transplant. 2007;13:1049-56.

9. Kim YJ, Li P, Hong JM, Ryu KH, Nam E, Chang MS. A Single center analysis of the positivity of hepatitis B antibody after Neonatal Vaccination Program in Korea. J Korean Med Sci. 2017;32:810-6.

10. Choung JM, Kim JC, Eun SH, Hwang PH, Nyhambat B, et al. Study on vaccination state in children : Jeonbuk province, 2000. Korean J Pediatr. 2002;45:1234-40.

11. Park S, Kim K, Kim DH, Jang JH, Kim SJ, Kim WS, et al. Changes of hepatitis B virus serologic status after allogeneic hematopoietic stem cell transplantation and impact of donor immunity on hepatitis B virus. Biol Blood Marrow Transplant. 2011;17:1630-7.

12. Li Volti S, Di Gregorio F, Romeo MA, Cannella A, Pizzarelli G, Sciacca A, et al. Immune status and the immune response to hepatitis $B$ virus vaccine in thalassemic patients after allogeneic bone marrow transplantation. Bone Marrow Transplant. 1997;19:157-60.

13. Shin HJ, Lee ES, Han SB, Lee JW, Chung NG, Cho B, et al. Serological changes against hepatitis B surface antigen in children and adolescents receiving chemotherapy for acute leukemia. Mediterr J Hematol Infect Dis. 2019;11:e2019052.
14. Lindemann $M$, Barsegian V, Runde V, Fiedler $M$, Heermann $\mathrm{KH}$ Schaefer UW, et al. Transfer of humoral and cellular hepatitis B immunity by allogeneic hematopoietic cell transplantation. Transplantation. 2003; 75:833-8.

15. Bonilla FA. Pharmacokinetics of immunoglobulin administered via intravenous or subcutaneous routes. Immunol Allergy Clin $\mathrm{N}$ Am. 2008;28:803-19.

16. Cerutti A, Puga I, Cols M. New helping friends for B cells. Eur J Immunol. 2012;42:1956-68

17. Abrahamsen IW, Sømme S, Heldal D, Egeland T, Kvale D, Tjønnfjord GE. Immune reconstitution after allogeneic stem cell transplantation: the impact of stem cell source and graft-versus-host disease. Haematologica. 2005;90:86-93.

18. Skert C, Damiani D, Michelutti A, Patriarca F, Arpinati M, Filì C et al. Kinetics of Th1/Th2 cytokines and lymphocyte subsets to predict chronic GVHD after allo-SCT: results of a prospective study. Bone Marrow Transplant. 2009;44:729-37.

19. Schillie S, Vellozzi C, Reingold A, Harris A, Haber P, Ward JW, et al Prevention of hepatitis B virus infection in the United States: Recommendations of the Advisory Committee on Immunization Practices. MMWR Recomm Rep. 2018;67:1-31.

20. Posuwan N, Vorayingyong A, Jaroonvanichkul V, Wasitthankasem R Wanlapakorn N, Vongpunsawad S, et al. Implementation of hepatitis $\mathrm{B}$ vaccine in high-risk young adults with waning immunity. PLoS One 2018;13:e0202637.

21. Inaba H, Hartford CM, Pei D, Posner MJ, Yang J, Hayden RT, et al Longitudinal analysis of antibody response to immunization in paediatric survivors after allogeneic haematopoietic stem cell transplantation. Br J Haematol. 2012;156:109-17.

22. D'Orsogna LJ, Wright MP, Krueger RG, McKinnon EJ, Buffery SI Witt CS, et al. Allogeneic hematopoietic stem cell transplantation recipients have defects of both switched and igm memory B cells. Biol Blood Marrow Transplant. 2009; 15:795-803.

23. Omazic B, Lundkvist I, Mattsson J, Permert J, Nasman-Bjork I Memory B lymphocytes determine repertoire oligoclonality early after haematopoietic stem cell transplantation. Clin Exp Immunol. 2003; 134:159-66.

24. Avanzini MA, Locatelli F, Dos Santos C, Maccario R, Lenta E, Oliveri M, et al. B lymphocyte reconstitution after hematopoietic stem cell transplantation: functional immaturity and slow recovery of memory CD27+ B cells. Exp Hematol. 2005;33:480-6. 